\title{
Waiting for Room Temperature Superconductivity
}

1. Evidence for superconductivity above $260 \mathrm{~K}$ in Lanthanum superhydride at megabar pressures

Authors: Maddury Somayazulu, Muhtar Ahart, Ajay K Mishra, Zachary M. Geballe, Maria Baldini, Yue Meng, Viktor V. Struzhkin, and Russell J. Hemley

arXiv:1808.07695

2. Superconductivity at $215 \mathrm{~K}$ in Lanthanum hydride at high pressures Authors: A. P. Drozdov, V. S. Minkov, S. P. Besedin, P. P. Kong, M. A. Kuzovnikov, D. A. Knyazev, M. I. Eremets

arXiv:1808.07039

\section{Potential high-Tc superconducting Lanthanum and Yttrium hydrides at} high pressure

Authors: Hanyu Liu, Ivan I. Naumov, Roald Hoffmann, N. W. Ashcroft, and Russell J. Hemley

Proc Natl Acad Sci U S A. 2017 Jul 3; 114(27): 69906995.

\section{Recommended with a Commentary by Chandra Varma, Visiting Scholar, Physics Department, New York University}

It has arrived - room temperature superconductivity or close enough to it. Reliable resistance measurements are reported by two groups on $\mathrm{LaH}_{10+x}$, at high pressures. $x$ is uncertain, estimated to be between -1 and 2. This appears to be a triumph of chemical synthetic techniques (for which I suggest the interested reader go to the first two papers above), as well as of the development of massive computational techniques using which, in the third paper above, (local) stability of the relevant fairly complicated crystal structures with well motivated atomic components was predicted, and reasonable estimates of its relevant electronic properties obtained.

The general physical basis itself is well understood and has a long history. Wigner and Huntington [1] in 1935, near the dawn of the understanding of quantum condensed matter and the metallic state, predicted that at sufficiently high pressure, insulating molecular hydrogen will transform to a metallic state of atomic hydrogen. Since the binding energy of $\mathrm{H}_{2}$ with respect to $2 \mathrm{H}$ is more than $4 \mathrm{eV}$, the pressure and the reduction of the volume of the metallic state has to be considerable. The best present estimates by density functional calculations [2] are about $500 \mathrm{GPa}$ or 5 Megabars. At such pressures, the inter-hydrogen 
distance is estimated to be about 1.15 Angstrom (while the intra-atomic distance in $H_{2}$ molecule is 0.75 Angstrom). The light mass and short inter-atomic distance leads to high vibration frequencies. The average phonon frequency $\langle\omega\rangle$ in the metallic state is estimated to be about $2300 \mathrm{~K}$ and the dimensionless electron-phonon coupling parameter $\lambda \approx 1.8$, with higher values at higher pressures. (The first estimates of such quantities [3] were made in jellium and Bohm-Staver approximations, which can be argued to be quite poor for the purpose and gave about 3,500 K and 0.45 respectively.) Superconductivity through electronphonon interactions, given the parameters of the metallic state, is a solved problem thanks to BCS in 1957. BCS has needed only small improvements made soon thereafter. Consider the BCS formula for weak-coupling superconductivity, $T_{c} \approx<\omega>e^{-1 / \lambda}$. With $<\omega>$ of about $2 \times 10^{3}$ degrees, a coupling constant $\lambda$ as small as $1 / 3$ gives room temperature superconductivity.

$500 \mathrm{GPa}$ is not easy to reach. (The pressure at the center of the earth is estimated to be about $380 \mathrm{GPa}$.) Measuring properties such as resistivity or better still magnetic susceptibility at such pressures is much harder. Although there have been reports of achieving metallic hydrogen over the years, no credible evidence for superconductivity was found. The closest one has come before the experiments reported in the two references above is squeezing on $\mathrm{H}_{2} \mathrm{~S}$, [4] which under pressures above $200 \mathrm{GPa}$ appears to transform partially to $\mathrm{H}_{3} \mathrm{~S}$, and shows superconductivity in resistivity measurements at about $200 \mathrm{~K}$. (See commentary by Fisk, Pickett and Thompson, JCCM-January-2017-02, for references on the experiments and subsequent calculations.) The superconducting volume fraction is unknown.

An even more clever recent idea is to form and squeeze on metal-hydrides. Bonding to the metal ion already squeezes the hydrogens to a higher density so that one may obtain a higher density at lower applied pressure. Using a variety of calculational techniques, the enthalpy of formation and local stability at various pressures for various metal hydrides in various possible structures was calculated. (1500 different structures were examined in many cases. The computer programs for these which are industrialized density functional calculations, with the charming name Quantum Espresso, are available online.) The one that has been synthesized was suggested in Ref. 3 above is $\mathrm{LaH}_{10}$, forms the sodalite structure, given in that paper, calculated to be stable above $200 \mathrm{GPa}$, with a inter-hydrogen distance of about 1.1 Angstrom.

The first paper above gives details of the very elaborate synthesis process which combines high pressure with laser heating. Evidence is provided that the contacts (four-probe) for resistance measurements stayed intact in the process. The predicted structure is found with about the predicted lattice constant. A fairly abrupt drop in resistance by more than a factor $10^{3}$ occurs at a temperature which varies with the pressure routine and samples with the highest at about $280 \mathrm{~K}$. Quite plausibly, this is the superconductivity temperature but no estimate of the superconducting fraction or critical currents is available. Presumably future measurements of the Meissner effect, etc. will provide much more information. The second paper, also combines high pressure with laser heating to form $\mathrm{LaH}_{10+x}$, with similar uncertainty in $x$, but has some differences in technique. It reports an abrupt drop in resistivity at a maximum of about $210 \mathrm{~K}$.

The electronic dispersion, the phonon dispersions and the electron-phonon interaction parameters are calculated in the third paper above using "Quantum Espresso" which uses one electron physics. This approach probably gives quite reasonable answers; electronic 
correlations may be un-important because of the high electronic density, $r_{S} \approx 1.5$. It would be better to actually calculating $\mu^{*}$, the electronic repulsion parameter in the pairing channel, for which only a very rough estimate is made. The calculated average phonon frequency and $\lambda$ are about $2,000 \mathrm{~K}$ and 2.2 respectively. Estimated $T_{c}$ from refinements of BCS theory is calculated with these parameters and the estimated $\mu^{*}$ to be about $270 \mathrm{~K}$. In the compound $\mathrm{YH}_{10}$, the calculations give a larger $\lambda$ and a much higher $\mathrm{T}_{c}$. No high pressure synthesis of this compound has been reported. The calculation of parameters through such programs is a black-box. No physical explanations for the range of coupling constants and their variation is given. We should expect a deeper understanding of the electronic and vibrational parameters in the metal-superhydrides and their variations in the future.

\section{References}

[1] E. Wigner and H. B. Huntington, J. Chem. Phys. 3, 1748 (1935).

[2] J. M. McMahon and D. Ceperley, Phys. Rev. B 84, 144515 (2011)

[3] N.W. Ashcroft, Phys. Rev. Lett., 21, 1748 (1968)

[4] A. P. Drozdov et al., Nature 525, 73 (2015). 